\title{
Three-dimensional nanonetworks for giant stretchability in dielectrics and conductors
}

Junyong Park', Shuodao Wang ${ }^{2,3}$, Ming Li ${ }^{4}$, Changui Ahn', Jerome K. Hyun', Dong Seok Kim', Do Kyung Kim', John A. Rogers ${ }^{5}$, Yonggang Huang ${ }^{2,3} \&$ Seokwoo Jeon ${ }^{1}$

The realization of levels of stretchability that extend beyond intrinsic limits of bulk materials is of great importance to stretchable electronics. Here we report large-area, three-dimensional nano-architectures that achieve this outcome in materials that offer both insulating and conductive properties. For the elastomer poly(dimethylsiloxane), such geometries enhance the stretchability and fracture strain by $\sim 62 \%$ and $~ 225 \%$ over the bulk, unstructured case. The underlying physics involves local rotations of narrow structural elements in the threedimensional network, as identified by mechanical modelling. To demonstrate the applications of three-dimensional poly(dimethylsiloxane), we create a stretchable conductor obtained by filling the interstitial regions with liquid metal. This stretchable composite shows extremely high electrical conductivity $\left(\sim 24,100 \mathrm{Scm}^{-1}\right)$ even at strains $>200 \%$, with good cyclic properties and with current-carrying capacities that are sufficient for interconnects in light-emitting diode systems. Collectively, these concepts provide new design opportunities for stretchable electronics.

\footnotetext{
${ }^{1}$ Department of Materials Science and Engineering, KAIST Institute for The Nanocentury, Korea Advanced Institute of Science and Technology (KAIST), Daejeon, 305-701, Republic of Korea. ${ }^{2}$ Department of Mechanical Engineering, Northwestern University, Evanston, Illinois 60208, USA. ${ }^{3}$ Department of Civil and Environmental Engineering, Northwestern University, Evanston, Illinois 60208, USA. ${ }^{4}$ State Key Laboratory of Structural Analysis for Industrial Equipment, Dalian University of Technology, Dalian 116024, China. ${ }^{5}$ Department of Materials Science and Engineering, University of Illinois at UrbanaChampaign, Urbana, Illinois 61801, USA. Correspondence and requests for materials should be addressed to S.J. (email: jeon39@kaist.ac.kr).
} 
S tretchable electronic materials enable classes of applications such as electronic eye cameras ${ }^{1}$, artificial skins ${ }^{2}$, flexible sensors ${ }^{3}$, and actuators ${ }^{4}$ that cannot be achieved using conventional, wafer-based technologies. One of the key technical challenges is in the development of materials that can offer the electronic properties of established inorganic materials but also can endure large-strain $(>1 \%)$, repeated deformations without changes in their characteristics. In most cases, the intrinsic material properties, such as yield strength, Young's modulus, Poisson's ratio, and so on, govern the mechanics. Recently, several approaches have been proposed to improve the stretchability beyond these limits; among these, two appear particularly promising. One exploits pre-stretching of a compliant substrate before bonding thin, stiff inorganic films, either uniformly or at strategic locations. Releasing the pre-strain leads to compression in the films and the corresponding formation of 'wavy' microstructures by the processes of buckling'. The other uses net-shaped flexible films with macroscopically perforated holes ${ }^{6}$. Stretchability of conductive elastomers can be extended up to $\sim 140 \%$ (ref. 7) by perforating, hot-rolling, and the use of a low elastic modulus substrate ${ }^{8}$. A disadvantage is that the stretching range in such two-dimensional structures is limited by the relatively low density of in-plane holes that can be accommodated, in a practical sense, at the millimeter scale.

Here we demonstrate a method that significantly improves the stretchability, and the range of design choices, by introducing perforations in full three-dimensional (3D) nanostructured forms, using a process that can easily be scaled to large areas $\left(\sim 625 \mathrm{~mm}^{2}\right.$ for results reported here). Such 3D net-shaped nanostructures in the elastomer poly(dimethylsiloxane) (PDMS) significantly improve the stretchability (by $\sim 62 \%$ ) compared with solid films made of the same material, owing to $3 \mathrm{D}$ rotations of elastic bridging elements when the structure is stretched. At the same time, the fracture strain is increased by more than $\sim 200 \%$. These enhancement mechanisms are identified quantitatively by theoretical modelling and verified by finite element analysis (FEA), both of which agree well with experimental results. To demonstrate the practical value of such strategies, we build highly stretchable conductors by infilling a liquid-phase eutectic gallium-indium (EGaIn) alloy into the interconnected, 3D porous channels in the structured PDMS. The composite results in extremely high electrical conductivity of $\sim 24,100 \mathrm{~S} \mathrm{~cm}^{-1}$ at $220 \%$ strain without severe deterioration during the many cycles of stretching-releasing. To the best of our knowledge, these values exceed those of other reported stretchable conductors. The 3D nano-architectures and the underlying mechanisms lead to new classes of stretchable materials, for potential use in various electronic and optoelectronic devices, as we demonstrate through interconnected arrays of light-emitting diodes (LEDs).

\section{Results}

Fabrication of 3D PDMS. Figure 1 illustrates a fabrication route to net-shaped 3D periodic elastomers that uses an optically patterned 3D nanostructure as a template. The process begins with 3D patterning of positive-tone photoresists by proximity-field nanopatterning $(\mathrm{PnP})^{9-12}$. PnP is an advanced 3D nanofabrication technique that involves a single-step exposure through a conformal phase mask. The mask used here involves surface relief structures consisting of cylindrical posts of diameter of $480 \mathrm{~nm}$, periodicity of $600 \mathrm{~nm}$, and height of $420 \mathrm{~nm}$ (Supplementary Fig. S1). Large-area, highly reliable fabrication of various hierarchical 3D nanostructures can be achieved by using large-size phase masks and the PnP technique. The whitish 3D nanostructure, shown in Fig. 1c (which, to date, corresponds to the largest 3D nanostructure patterned by $\mathrm{PnP}$ ), serves as a template to produce the $3 \mathrm{D}$ elastomer. A key advance over previous work is the replacement of conventional $3 \mathrm{D}$ resists (e.q. $\mathrm{SU}-8$, Microchem $)^{13}$, which require harsh removal conditions such as high-temperature burning $\left(\sim 500{ }^{\circ} \mathrm{C}\right)$ or plasma etching ${ }^{14}$, with a positive-tone resist (AZ 9260, Clariant). The positive-tone resist can be easily removed by buffered aqueous solution of $\mathrm{KOH}$ after infiltration of PDMS, without causing damage to the 3D nano-architecture. When the 3D structure is filled with PDMS, it becomes transparent (Fig. 1d) owing to approximate index matching between AZ 9260 and PDMS (refractive indices $\sim 1.6$ and $\sim 1.4$, respectively). A second flood exposure renders the original template and the releasing layer (hard-baked AZ 9260) soluble by a developer (AZ $400 \mathrm{~K}, 1: 4$ dilution of buffered $\mathrm{KOH}$, Clariant). The 3D nanostructured PDMS film with a residual layer of solid PDMS (thickness of the residual layer varies with different preparation condition; Supplementary Fig. S2) can then be released from the glass supporting substrate into the developer. A thick slab of PDMS can be used to retrieve the resulting free-standing sheet of 3D PDMS (Fig. 1e,f).

Most positive-tone resists have high absorption coefficients, which restricts the maximum thicknesses that can be utilized in the PnP process to $\sim 3 \mu \mathrm{m}$ (refs 15,16 ). This limitation can be overcome by the unique optical properties of DNQ-based resists. In particular, this resist system chemically converts into indene-carboxylic acid photoproducts under ultraviolet light, resulting in reduced absorption with increasing exposure time (that is, photo-bleaching effect) ${ }^{17}$ (Fig. 2a). Accordingly, the achievable thicknesses of 3D patterns increase with exposure dose. However, we note that the use of large doses may result in overexposure at the top of the resist. During development, this can lead to damaged $3 \mathrm{D}$ structures near the top and a porosity gradient along the ultraviolet penetration depth. To avoid these problems, exposure time, developing time and resist thickness must be carefully controlled. The structures that result after development become more opaque with increasing exposure dose, because the 3D structure scatters more light as the thickness of the patterned structure increases (Fig. 2b) ${ }^{10}$. Cross-sectional scanning electron microscope (SEM) images confirm this effect (Fig. 2c, d). Thick $3 \mathrm{D}$ structures $(\sim 12 \mu \mathrm{m})$, containing periodic multilayers, can be fabricated at the exposure dose of $\sim 450 \mathrm{~mJ} \mathrm{~cm}^{-2}$. Measured values of the extinction coefficients as a function of dose (Supplementary Fig. S3) and the corresponding light propagation analysed by FEA further explain the phenomena (Supplementary Fig. S4). Over-developing exposes the surfaces of the 3D structures, to reveal successful inner-layer patterning, with results that are consistent with electromagnetic simulations using GSolver (Grating Solver Development) (Fig. 2e,f). Figure $2 \mathrm{~g}$ shows easy removal of the patterned AZ 9260 by a water-based developer, which does not swell, dissolve or distort soft organic materials such as PDMS ${ }^{18}$ (Supplementary Fig. S5). Taken together, these properties make this type of resist ideal for present purposes.

Highly stretchable 3D PDMS. Vacuum-assisted infiltration provides a route to complete filling of PDMS prepolymer into the sub-micron pores of the $3 \mathrm{D}$ templates ${ }^{15}$. Spin-casting follows the infiltration to flatten the surface of the sample. The speed of spincasting (1,000 to 7,000 r.p.m.) controls the residual thickness of solid PDMS (portion of the prepolymer outside the 3D template, on top). In the following, the thickness of the 3D PDMS is fixed at $12 \mu \mathrm{m}$, while the total film thickness ranges from 15 to $90 \mu \mathrm{m}$ (that is, residual solid layer is $3 \sim 78 \mu \mathrm{m}$; Supplementary Fig. S6). The curing temperature of PDMS was fixed at $60^{\circ} \mathrm{C}$ to ensure sufficient crosslinking of polymer chains. Relatively thin 3D PDMS, inversely replicated from the template, experiences severe bending forces because of its high porosity and osmotic pressure during drying immediately after retrieval from the developer. Conformal backing layers of unpatterned PDMS provide mechanical support, for manipulation in a way that minimizes unwanted deformations (Fig. 3a). In this way, the $3 \mathrm{D}$ PDMS maintains its open porous structures without any distortion or collapse of the interconnected features. The coverage of $3 \mathrm{D}$ structures is not limited to bulk volumes, but can also be 
a
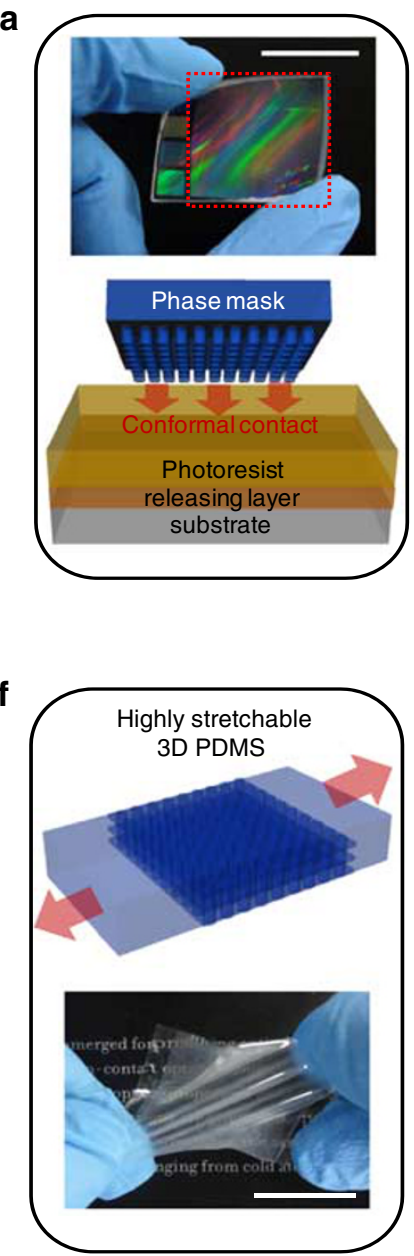
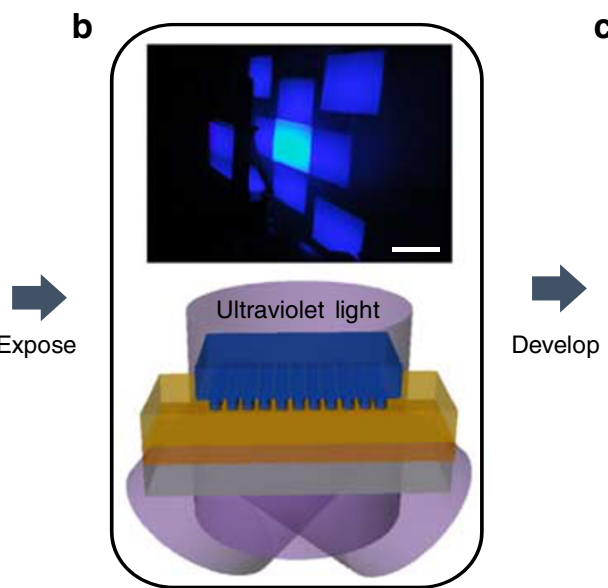

c

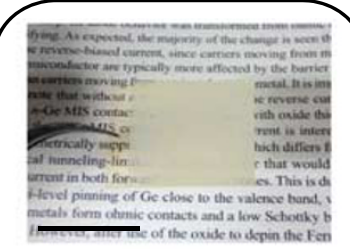

Wits form chimic contacts and a lew Schooky b

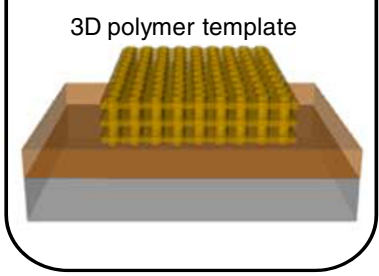

PDMS infiltrate \& overexpose
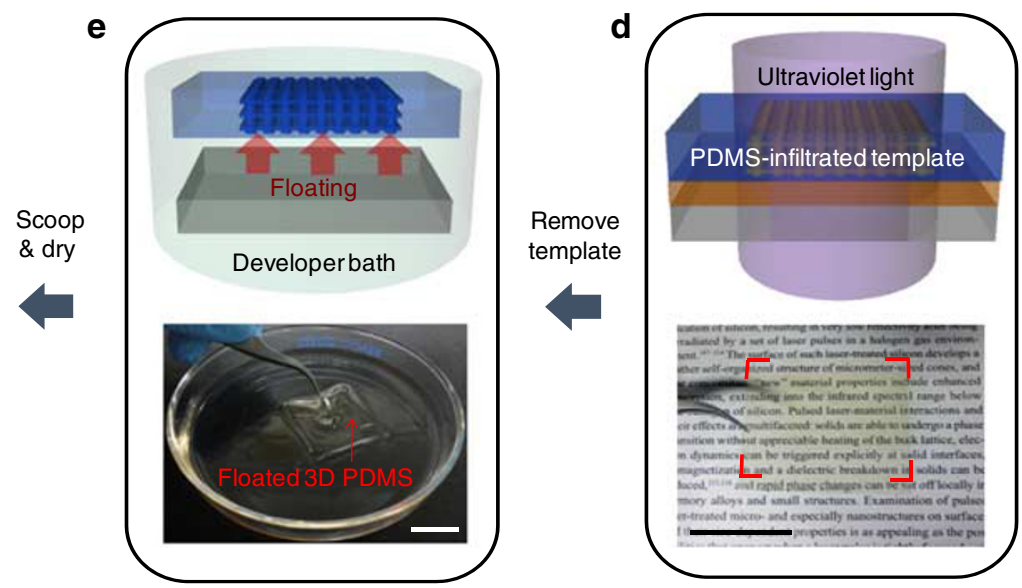

Figure 1 | Schematic illustration of fabrication procedures to produce 3D PDMS. (a) Conformal contact of a PDMS phase mask against a film of a positive-tone photoresist. (b) Generation of complex 3D distributions of intensity in the photoresist by interference of ultraviolet light (wavelength $\sim 355 \mathrm{~nm}$ ) diffracted by passage through the mask. (c) Washing away the exposed soluble regions using water-based developing solution. (d) Infiltration of PDMS prepolymer into the 3D template, followed by a second flood exposure. (e) Removal of the 3D template and releasing layer (hard-baked photoresist) in a developer bath. (f) Retrieving the floating PDMS membrane and drying it with a stream of air. Scale bar, $2 \mathrm{~cm}$.

realized as line or arbitrary shapes by using a superimposed multiple-mask system or performing additional patterning (Supplementary Fig. S7) ${ }^{19-20}$. The optical image of a folded 3D PDMS film with line patterns is shown in Fig. 3b. Figure $3 \mathrm{c}$ shows a top-view SEM image of 3D PDMS.

The resulting 3D nano-architectures in PDMS offer superior levels of stretchability. We identify two important features of the mechanics from the experimental results (Fig. 3d). The first is that PDMS films with 3D nanostructures exhibit fracture strains significantly higher than those of solid PDMS films with the same thickness. In other words, 3D periodic networks enhance the fracture limits. The second is that 3D PDMS films show systematically different mechanical behaviour as the overlying solid film thickness decreases. In particular, the 3D PDMS shows increasing fracture strain with decreasing film thickness, whereas solid PDMS typically shows the opposite trend ${ }^{21}$. The stress-strain curves support these experimental results (Supplementary Fig. S8). Once the 3D PDMS reaches breaking point, fracture occurs abruptly. The level of enhancement in stretchability (compared with solid PDMS) significantly increases as the total film thickness (Fig. 3e) decreases by reducing the overlayer thickness, that is, as the ratio $R$ of the thickness of the $3 \mathrm{D}$ layer to that of the solid layer varies from 0.15 to 4 . This effect becomes increasingly dominant as $R$ increases. When $R$ is low $(<0.2)$, the elastic modulus of 3D PDMS is similar to that of solid PDMS. As $R$ increases, the elastic modulus of 3D PDMS is significantly lower than that of solid PDMS at the same film thickness. When $R$ is greater than 1, the elastic modulus of 3D PDMS is $\sim 22 \%$ of that of solid PDMS.

The curing temperature also influences the mechanical properties, because the degree of PDMS vulcanization heavily depends on this temperature (Supplementary Fig. S9). Elastomers typically have high flexibility and stretchability when cured at low temperatures ${ }^{22}$. The 3D PDMS cured at room temperature shows maximum stretchability of $\sim 225 \%$, which corresponds to the highest value reported for PDMS (Supplementary Movie S1 for the stretching test of solid PDMS and solid PDMS).

Enhancement mechanism. An analytical mechanics model, validated by FEA, is developed to identify the mechanism of these enhancements. Although mechanical simulations for large compressive deformation in $2 \mathrm{D}$-ordered porous polymers have been studied $^{23}$, deformation mechanics of 3D-ordered porous elastomers under stretching is not well-known. The symmetry of 3D polymer structure fabricated from $\mathrm{PnP}$ is similar to body-centred tetragonal with sub-micron lattice parameters ${ }^{20}$. The shape and geometry of resulting 3D PDMS structures take the inverse form of the original 


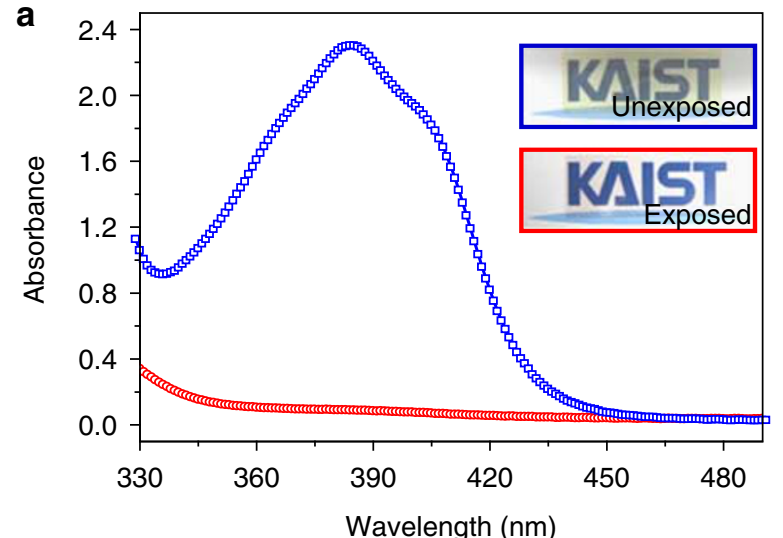

b
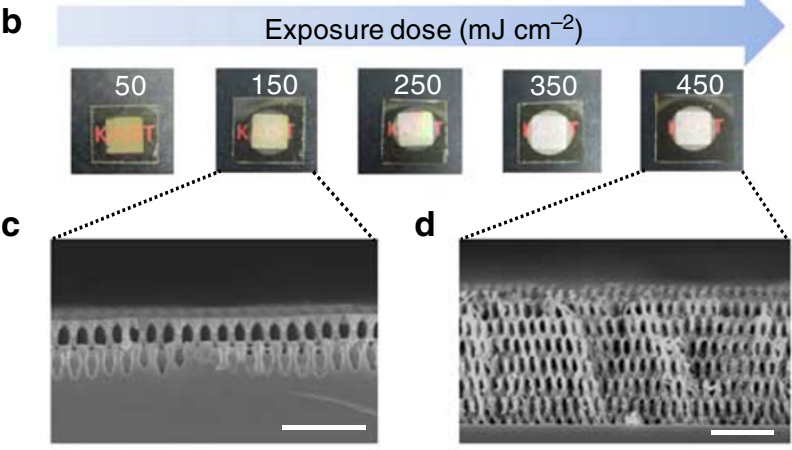

e

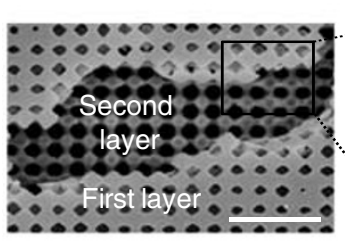

$\mathbf{f}$

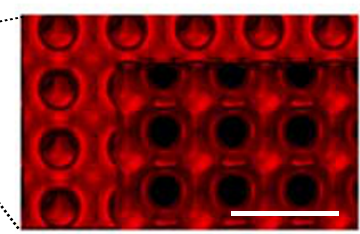

g

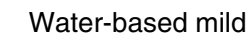
solution (AZ 400K)
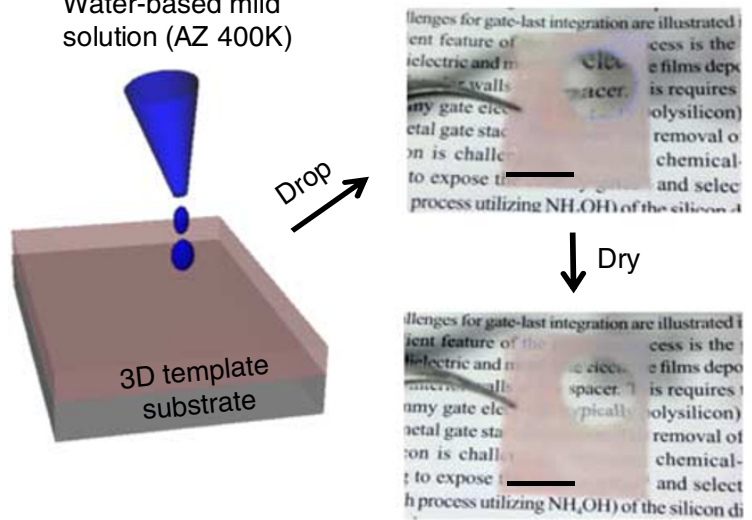

Figure $\mathbf{2}$ | Fabrication of 3D periodic templates using thick positivetone photoresists. (a) Reduction in optical absorption in the DNQ-based photoresist film (AZ 9260, Clariant) induced by photoexposure (Blue open squares: unexposed photoresist film, red open circles: exposed photoresist film). (b) Change in transparency of 3D patterned films with increasing exposure dose. (c) Cross-sectional SEM image of a AZ 9260 film patterned using an exposure dose of $150 \mathrm{~mJ} \mathrm{~cm}^{-2}$. Scale bar (also in d,e), $3 \mu \mathrm{m}$. (d) Cross-sectional SEM image of a AZ 9260 film patterned using the exposure dose of $\sim 450 \mathrm{~mJ} \mathrm{~cm}^{-2}$. (e) Top-view SEM image of the top surface of the 3D polymer template after removing part of the first layer. (f) Optical simulations of expected 3D structures, performed by rigorous coupled wave analysis. Scale bar $1 \mu \mathrm{m}$. (g) Removal of AZ 9260 templates by acqueous basic solution (AZ $400 \mathrm{~K}$ developer, Clariant). Scale bar, $1 \mathrm{~cm}$. template (see Supplementary Fig. S10 for detailed visualizations and optical simulations on the geometry). As a result, a unit cell of the 3D PDMS consists of ellipsoids (prolate spheroid) with polar radius $A$ and equatorial radius $B$, respectively (Supplementary Fig. S11). The ellipsoids are packed in a body-centred tetragonal Bravais lattice with square base $2 W \times 2 W$ and height $2 H$, and interconnected with bridging elements of length $L$. The length $L$ is defined as the surface-to-surface distance between two adjacent ellipsoids along the line segment between their origins, and is related to the above geometric parameters by

$$
L=\sqrt{2 W^{2}+H^{2}}-2 \sqrt{\left(1+\frac{2 W^{2}}{H^{2}}\right) /\left(\frac{1}{A^{2}}+\frac{2 W^{2}}{B^{2} H^{2}}\right)}
$$

The projection of $L$ into the stretching direction ( $y$ axis, see Supplementary Fig. S11 for direction definitions) is $l$, as shown in Fig. 3f (where everything is projected into the $y-z$ plane). When the 3D PDMS is stretched, the bridging elements first rotate to the direction of stretching, to accommodate the deformation, which offers an longation of $2 \cdot(L-l)$ for each unit cell. The strain at this stage mainly involves bending strain, which is small compared with the large stretching that follows. Further stretching mainly appears in the bridging elements, because they have much smaller crosssectional area (and thus smaller tensile stiffness) than the ellipsoids. Each bridging element can be stretched to a length of $L \cdot\left(1+\varepsilon_{\mathrm{s}}\right)$ before fracture occurs, where the $\varepsilon_{\mathrm{S}}$ is the fracture strain for solid PDMS. Therefore, the fracture strain for $3 \mathrm{D}$ PDMS is

$\varepsilon_{3 \mathrm{D}}=\frac{L\left(1+\varepsilon_{\mathrm{s}}\right)-l}{W}=\left(1-2 / \sqrt{\frac{H^{2}}{A^{2}}+\frac{2 W^{2}}{B^{2}}}\right)\left[\left(1+\varepsilon_{\mathrm{s}}\right) \cdot \sqrt{2+\frac{H^{2}}{W^{2}}}-1\right]$

This simple model shows that slender unit cell (large $H / W$ ) and relatively small ellipsoids (large $H / A$ and $H / B$, or the structure being more porous) enhance the stretchability of the $3 \mathrm{D} \mathrm{PDMS}^{7}$. For experimental data of $W=250 \mathrm{~nm}, H=950 \mathrm{~nm}, A=500 \mathrm{~nm}, B=200 \mathrm{~nm}$, and $\varepsilon_{\mathrm{s}}=85 \%$ (Fig. $3 \mathrm{~d}$, solid PDMS made at the spin-speed of 7,000 r.p.m.), equation (2) gives $\varepsilon_{3 \mathrm{D}}=149 \%$, which agrees well with experimental result of $147 \%$ (Fig. $3 \mathrm{~d}$ ). For, $\varepsilon_{\mathrm{s}}=160 \%$, obtained by curing at room temperature, equation (2) gives $\varepsilon_{3 \mathrm{D}}=219 \%$, which is consistent with experimental result of $225 \%$. Fig. $3 \mathrm{~g}$ shows FEA results for 3D PDMS under stretching. For $90 \%$ strain, the bridging elements rotate to the stretching direction to accommodate the deformation, resulting in strain of only about $20 \%$ in the bridging elements; further stretching to $150 \%$ significantly increases the strain in the bridging elements to about $70 \%$. During the entire stretching, the bridging elements rotate and stretch to accommodate almost all of the deformation, while the strain in the ellipsoids remains small. The FEA results in Fig. $3 \mathrm{~g}$ also show that $150 \%$ stretch induces a uniform true strain of $92 \%$ in the solid PDMS, while the 3D PDMS has strain larger than $90 \%$ only in very small, localized regions (small, red area in Fig. 3h). Consequently, the statistically possible number of fracture points in 3D PDMS during stretching is significantly fewer than that of solid PDMS.

High-performance 3D stretchable conductor. The high stretchability of 3D PDMS makes it an attractive alternative to solid PDMS as an insulating substrate in stretchable electronics, and potentially as interlayer dielectrics as well. Furthermore, the open spaces can be exploited to achieve composite materials with distinct electronic properties $^{24}$. For example, 3D PDMS can be made electrically conductive by filling conductive materials into the continuous, interconnected channels of its $3 \mathrm{D}$ pores. A stretchable conductor, which 


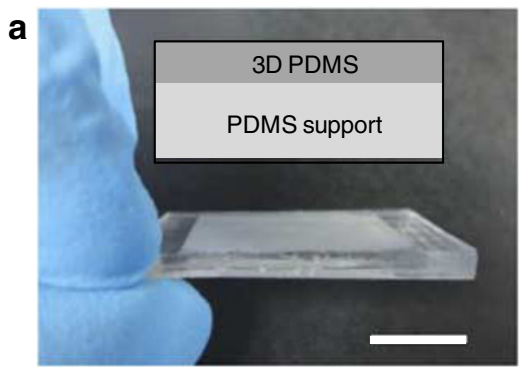

b

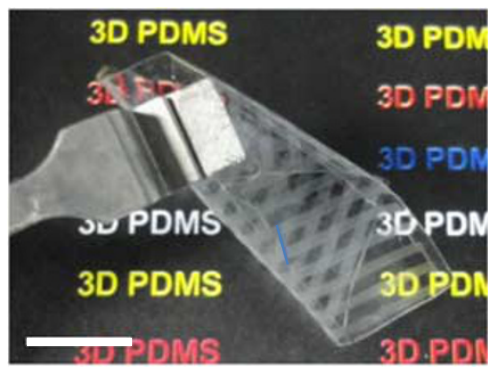

c

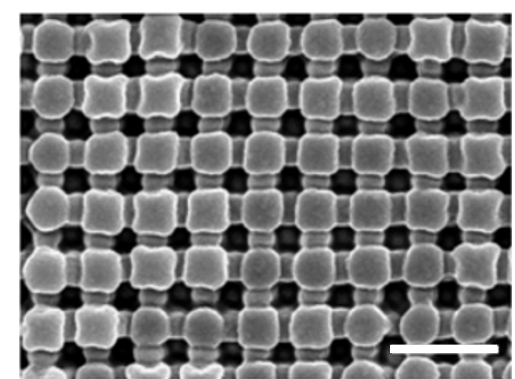

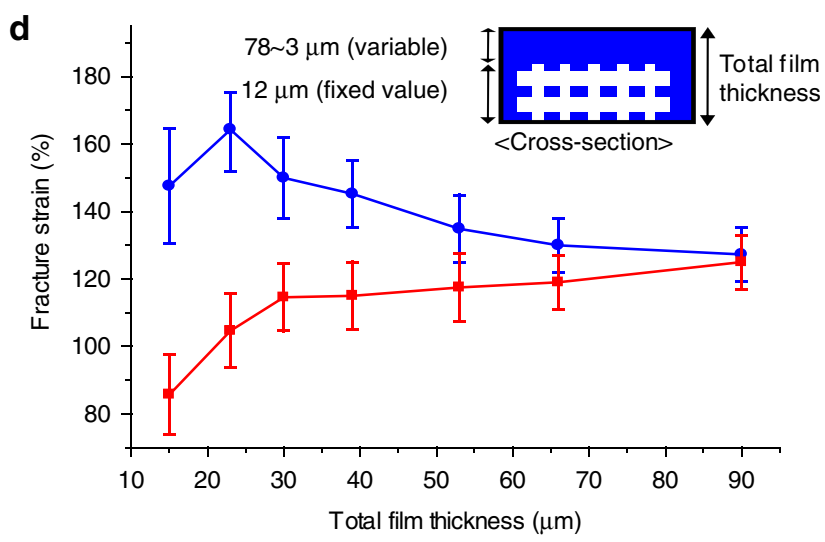

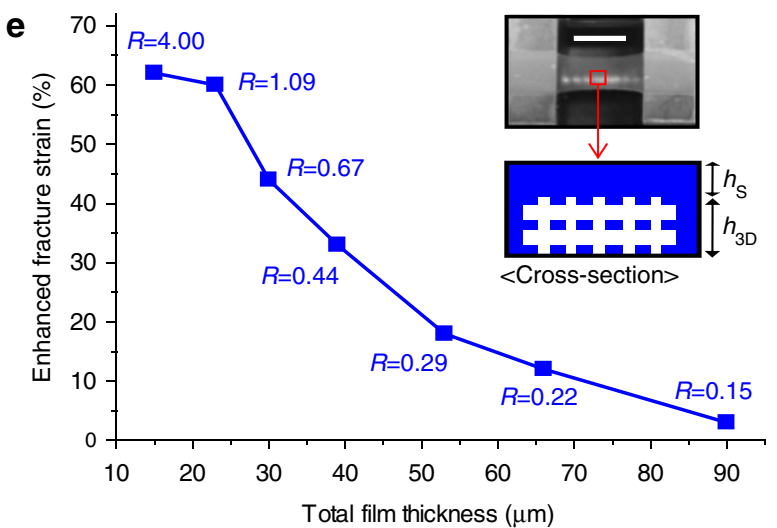

$\mathbf{f}$
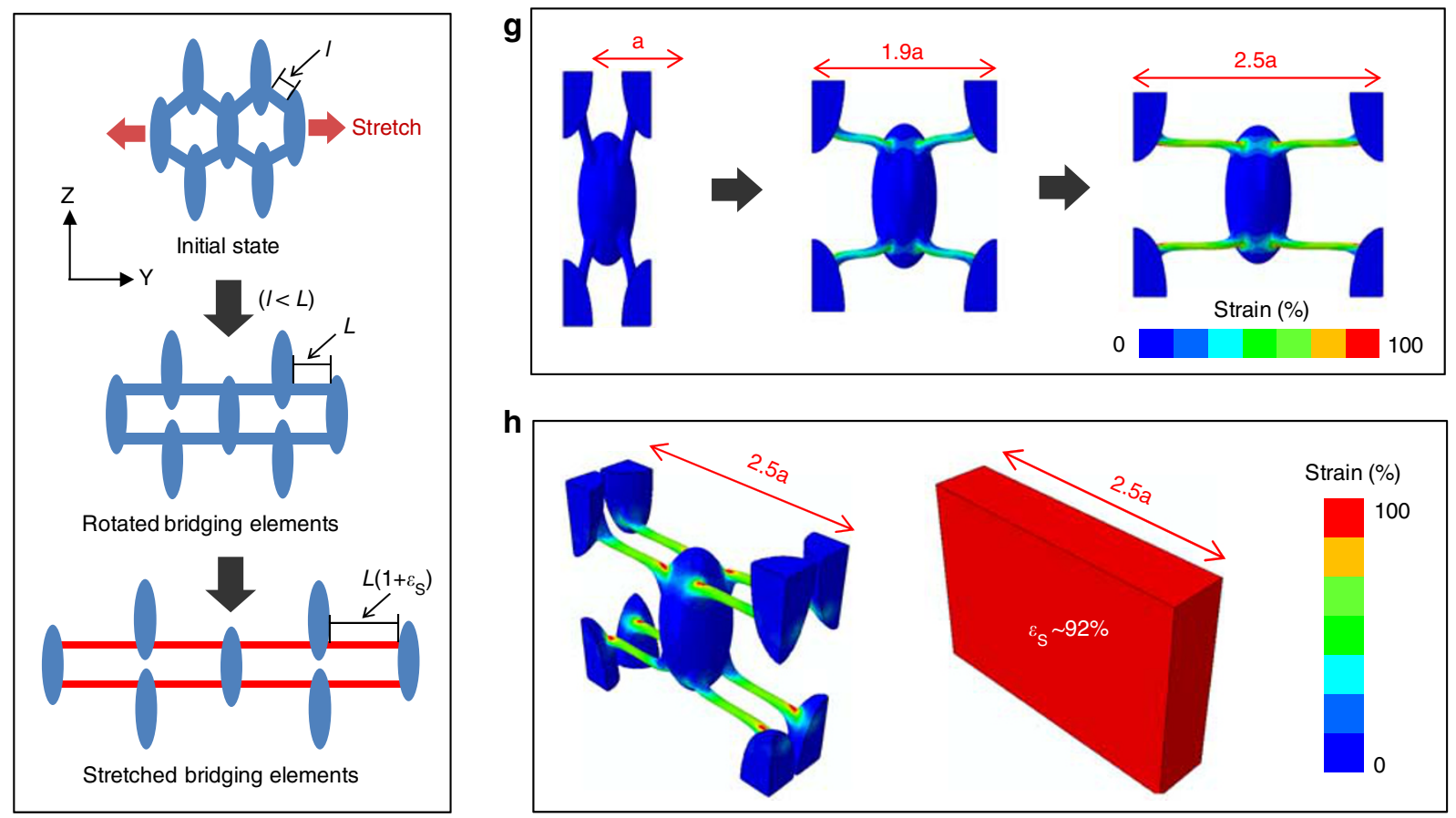

Figure 3 | Mechanical properties of 3D PDMS. (a) Optical image of a supported 3D PDMS film. Scale bar, $1 \mathrm{~cm}$. (b) Optical image of a folded 3D PDMS film with line patterns. Scale bar, $1 \mathrm{~cm}$. (c) Top-view SEM image of net-shaped 3D PDMS. Scale bar, $1 \mu \mathrm{m}$. (d) Comparison of fracture strain between 3D PDMS (blue-filled circles) and solid PDMS (red-filled squares) for various thicknesses. (e) Comparison of extended stretchability for different ratios of thickness of the 3D layer to that of the solid film $\left(R=h_{3 D} / h_{\mathrm{s}}\right)$. Inset: optical image of 3D PDMS attached on the customized tensile tester. Scale bar, $1 \mathrm{~cm}$. (f) Rotation mechanism of the 3D PDMS under stretching along Y-direction, projected in the Y-Z plane. (g) Strain distribution obtained by FEA for the unit cell of 3D PDMS stretched by $90 \%$ (middle) and 150\% (right). (h) Comparison of strain distributions between 3D PDMS (left) and solid PDMS (right) under $150 \%$ stretching.

consists of liquid metal (EGaIn) enclosed in the elastomeric channels at room temperature, is created by sandwiching two thin $(\sim 23$ $\mu \mathrm{m})$ 3D PDMS films. The EGaIn can be smoothly injected into 3D channels along the plasma-treated surfaces with vacuum-assistance.
The colour of EGaIn is changed from bright silver to whitish gray owing to light scattering after successful filling. Figure 4 summarizes the electrical properties of the resulting composite and its practical demonstration in a simple connection of LEDs. This system 


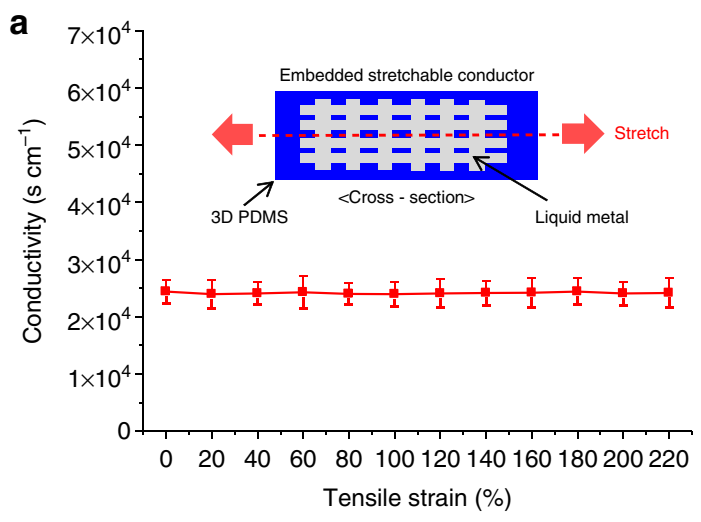

C

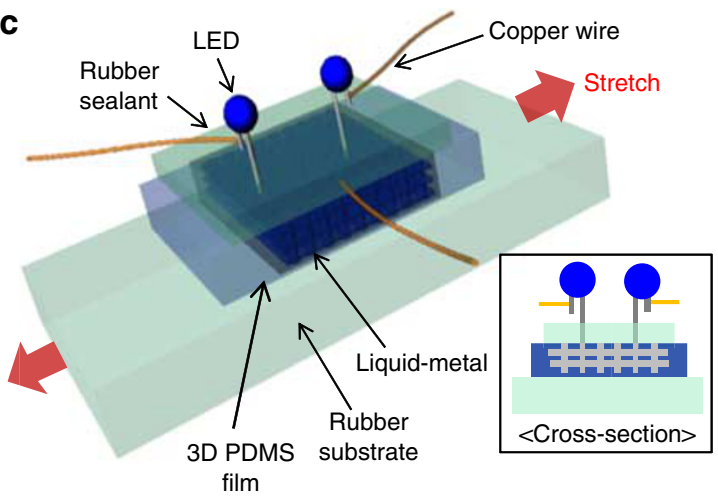

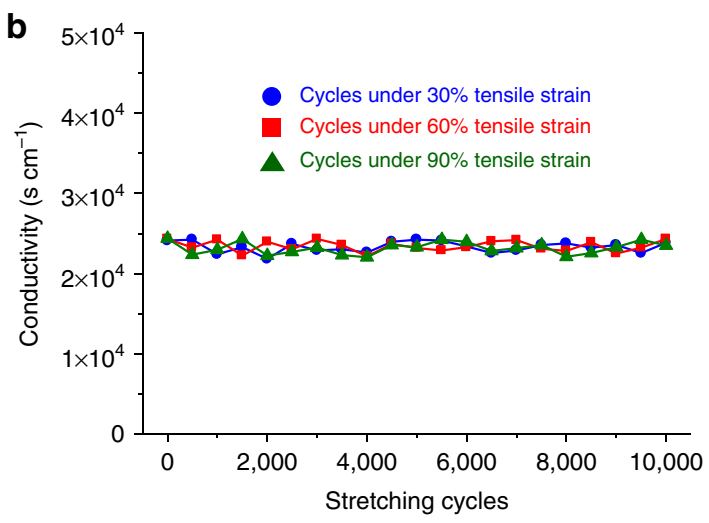

d
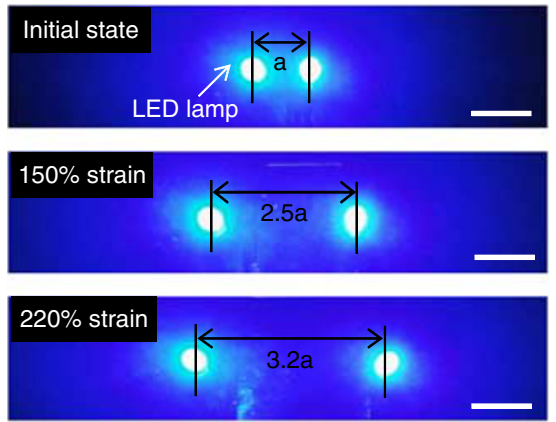

e

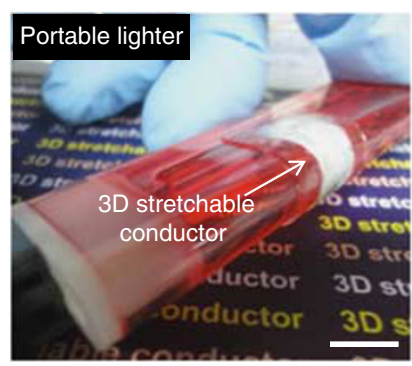

f

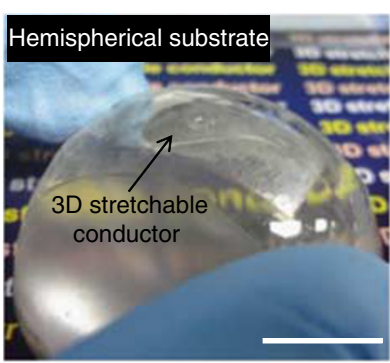

g

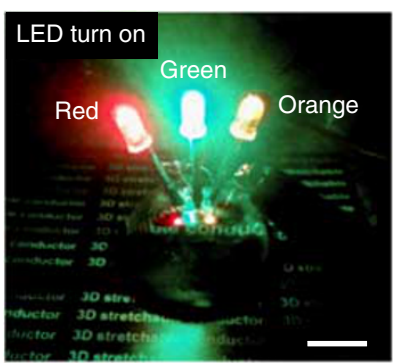

Figure 4 | Electrical robustness of 3D stretchable conductor and its demonstration through LED applications. (a) Conductivity of embedded stretchable conductors under stretching up to $220 \%$. (b) Conductivity after cyclic stretching-releasing of various strains. (c) Schematic illustration of LED devices on stretchable conductors. (d) Successful operation under stretching up to $220 \%$. (e) Conformally attached stretchable conductors on the portable lighter. (f) Conformally attached stretchable conductors on a hemispherical substrate ( $g$ ) Realization of LED arrays (Red, green, orange colours) using 3D stretchable conductors on the hemispherical substrate (f). Scale bar, $1 \mathrm{~cm}$.

shows extremely high electrical conductivity $\left(\sim 24,100 \mathrm{Scm}^{-1}\right)$, comparable to the intrinsic conductivity of EGaIn. The conductivity is invariant during the entire stretching process, owing to the ability of the fluid-phase EGaIn to follow any changes in the shape of the stretched 3D PDMS, and maintain continuous conduction pathways through the porous network (Fig. 4a) (Supplementary Fig. S12a for the conductance change under stretching). The conductivity of $\sim 24,100 \mathrm{~S} \mathrm{~cm}^{-1}$ at $220 \%$ strain represent improvements on previous results for stretchable conductors (Supplementary Fig. S12b). The slight decrease in stretchability ( 5\%) compared with non-conductive 3D PDMS results from the increased portions of solid PDMS around the perimeter, for sealing the EGaIn. Cyclic tensile tests at various strains are shown in Fig. 4b. The conductivity remains nearly the same even after 10,000 cycles of $90 \%$ strain, thereby demonstrating the mechanical robustness of this system. This strategy overcomes major weakness of conventional stretchable conductors, which have a trade-off relationship between stretchability and conductivity ${ }^{25}$.
The potential applications for 3D stretchable conductors include stretchable antennas, wearable keypads and flexible sensors ${ }^{26-31}$. We demonstrate a simple prototype that consists of commercial LED lamps integrated with and interconnected by the $3 \mathrm{D}$ stretchable conductor. Figure $4 \mathrm{c}$ shows a schematic illustration of the setup (see Supplementary Fig. S13 for the real image of the set-up on stretching tester). Two LED lamps are installed near opposite ends of the stretchable conducting layer, and connected through the 3D stretchable conducting layer. The LED lamps are turned on when the applied voltage is greater than $2.3 \mathrm{~V}$ (Supplementary Fig. S14) and survive after stretching up to $\sim 220 \%$ (Fig. $4 \mathrm{~d}$ ). The brightness does not degrade even beyond $\sim 220 \%$ because of the electrical and mechanical robustness of embedded stretchable conductor. The illumination power of a LED lamp integrated into the 3D stretchable conductor is comparable to that of a system employing gold electrodes at $0 \%$ strain (Supplementary Fig. S15). We also show that the conductive path can be maintained on non-planar substrates (Fig. 4e,f), where conformal wrapping can occur easily owing to 
the low-bending modulus, thin form, and highly elastic properties. LED arrays with various colours (red, green, orange) can be realized on hemispherical substrates using this approach (Fig. 4g).

\section{Discussion}

The methods and the collective set of results reported here represent concepts for realizing materials and structures with much higher levels of stretchability and conductivity compared with conventional methods. A critical component to this work is the identification of the mechanism responsible for the enhanced stretchability beyond intrinsic limits in 3D nanostructured materials. The strategy is agnostic to the particular elastomer formulation. The most important outcome, then, is the improvement in stretchability for the elastomer studies, rather than an absolute comparison against other materials. A remaining technical barrier in application of the technology is the fabrication of thicker 3D structures. Exploring other materials, such as metal or ceramic, represents another promising direction for future work.

\section{Methods}

Preparation of silicon masters. A bottom antireflective coating (K-131, Dongin Semichem) with a thickness of $\sim 58 \mathrm{~nm}$ was formed on an 8-inch silicon wafer using a track system (K Spin8, SEMES). Then, a thin photoresist (LX-429, Dongjin Semichem) film with a thickness of $\sim 400 \mathrm{~nm}$ was spincoated and exposed at $22 \mathrm{~mJ} \mathrm{~cm}^{-2}$ through a pre-patterned ultraviolet reticle using a $\mathrm{KrF}(248 \mathrm{~nm})$ scanner (NSR-S203B, Nikon). After developing the exposed film with developer (DPD 200 , Dongjin Semichem) for 60 s, bottom antireflective coating etching was performed with a deep silicon etcher (TCP-9400, Lam Research) using CF $_{4}$ gas and a flow rate of 100 s.c.c.m., at a main power of $250 \mathrm{~W}$ with a bottom power of $-250 \mathrm{~W}$, at 7 mTorr. Subsequently, silicon etching formed relief structures with depths of $\sim 400 \mathrm{~nm}$, using $\mathrm{Cl}_{2}$ gas with a flow rate of 72 s.c.c.m., $\mathrm{HBr}$ gas with a flow rate of 180 s.c.c.m., $\mathrm{O}_{2}$ gas with a flow rate of 7 s.c.c.m., at a main power of $300 \mathrm{~W}$ with a bottom power of $-225 \mathrm{~W}$. Finally, the residual photoresist on the top of silicon masters was removed using a photoresist stripper (DAS2000, PSK).

Preparation of conformal phase masks. The silicon master was pre-treated by perfluorinated trichlorosilane (T2492, United Chemical Technologies) vapour in a dessicator to prevent strong adhesion between the master and the silicone elastomer. A bilayer structure of two different types of PDMS was used to replicate nanostructures against the master. A hard modulus PDMS ( 10 MPa) (h-PDMS) (VDT-731, HMS-301, Gelest) was directly spin-coated onto the master at 1,000 r.p.m. for 30 s. Next, continuous spinning at 500 r.p.m. for 30 min yielded partially crosslinked h-PDMS with a flat surface. Subsequently, a relatively soft PDMS ( 2 MPa) (s-PDMS) (Sylgard 184, Dow Corning) was poured on top of the first layer to facilitate manipulation of the resulting soft, conformable phase mask. Fully curing the bilayer PDMS for 1 day at room temperature and then peeling it away from the master completed the process.

Preparation of photoresists on substrates. A glass substrate (G480-15, ProSciTech) was oxygen-plasma treated with a flow rate of 45 s.c.c.m., a pressure of $40 \mathrm{mTorr}$, and a power of $60 \mathrm{~W}$ for $2 \mathrm{~min}$ using a plasma treatment system (CUTEMP, Femtoscience). Thin AZ $9260(\sim 5 \mu \mathrm{m})$ was pre-coated on the cleaned substrate as a releasing layer to enable delamination of free-standing thin films of PDMS from the substrate during the template removal step. The releasing layer was hardbaked on at $110^{\circ} \mathrm{C}$ for $5 \mathrm{~min}$. Subsequently, relatively thick photoresist $(\sim 12 \mu \mathrm{m})$ was spin-coated on the releasing layer at a spin-speed of 2,000 r.p.m. for 30 s. The resist-coated substrate was then soft-baked at $100^{\circ} \mathrm{C}$ for $5 \mathrm{~min}$.

Fabrication of 3D templates by Proximity-field nanoPatterning (PnP). The optical set-up for PnP includes a pulsed, passively Q-switched Nd:YAG microchip laser $(355 \mathrm{~nm}, 14 \mathrm{~mW}$, Teem Photonics) with a beam expander and a collimator as a coherent ultraviolet light source. Placing the PDMS phase mask on the surface of the AZ 9260 film leads to intimate atomic scale, conformal contact driven by Van der Waals forces without any pressure. Passing collimated light through the mask generates a complex 3D intensity distribution in the AZ 9260 film. The exposure doses ranged from 50 to $450 \mathrm{~mJ} \mathrm{~cm}^{-2}$. The exposed regions were then dissolved away using a water-based developer (AZ $400 \mathrm{~K}, 1: 3$ dilution of buffered $\mathrm{KOH}$, Clariant) and rinsed by de-ionized water.

Fabrication of 3D PDMS by templating processes. A s-PDMS prepolymer was prepared by mixing the base component and the curing agent in 10:1 ratio. The mixture was degassed in a small dessicator for $1 \mathrm{~h}$ to completely remove air bubbles, and it was poured onto the oxygen-plasma treated $3 \mathrm{D}$ template. Infiltrated sample was degassed again in a dark dessicator for $1 \mathrm{~h}$ to ensure that prepolymer successfully filled into the porous nanonetwork of the 3D template. Spin-casting with various spin-speeds ranging from 1,000 to 7,000 r.p.m. enabled a controlled process for levelling an upper residual layer consisting of solid PDMS. After curing the s-PDMS at $60{ }^{\circ} \mathrm{C}$ for $2 \mathrm{~h}$ in a clean oven, a second flood exposure allowed the original template and the releasing layer to be removed with organic solvents. A free-standing PDMS film, which was inversely replicated from the 3D polymer template, floated onto a developer bath (AZ $400 \mathrm{~K}, 1: 4$ dilution of buffered $\mathrm{KOH}$, Clariant) as a result of dissolving the releasing layer. Scooping up this film using a thick, conformal PDMS slab successfully yielded a back-supported thin, 3D porous sheet of PDMS

Fabrication of stretchable conductors. The stretchable conductor was prepared by infiltrating eutectic gallium-indium alloy (EGaIn, Sigma-Aldrich) into 3D PDMS. Two samples of 3D PDMS with narrow strips of solid PDMS at the edges, supported by thick PDMS slabs with releasing layers (AZ 5214-E, Clariant), were oxygen-plasma treated at a flow rate of 45 s.c.c.m., pressure of 40 mTorr, and power of $60 \mathrm{~W}$ for $120 \mathrm{~s}$. EGaIn was dropped and spread on the top of the plasma-treated 3D PDMS. The sample was degassed in a dessicator overnight to squeeze EGaIn into the porous channel of 3D PDMS. After pressing on the top of EGaIn-filled 3D PDMS with a PDMS block and the sample was spun-cast at 8,000 r.p.m. to reduce residual EGaIn remaining outside the pores. To ensure successful filling, the above infiltration steps were repeated several times. Finally, two samples prepared with this method were sealed together. Weakly pressing the marginally solid PDMS for few seconds enables perfect sealing of EGaIn. Removal of releasing layers by developer yielded enclosed stretchable conductors.

Optical modelling. The 2D calculation of the intensity distribution of the ultraviolet light in the photoresist film, through the PDMS phase mask, was conducted using a finite elements modelling software (COMSOL Multiphysic 3.5, COMSOL). The 3D reconstructed images of the structures formed by PnP were simulated using rigorous coupled wave analysis software (GSOLVER V4.20, Grating Solver Development)

Characterization. The structural details of positive-tone templates and 3D PDMS were investigated by field-emission scanning electron microscopy (S-4800, Hitachi) operated at an accelerating voltage of $5-10 \mathrm{kV}$. Absorption measurements of $\mathrm{AZ}$ 9260 films were performed using a UV-VIS spectrometer (UV-310PC, Shimadzu). The mechanical tensile tests were conducted using a customized flexible tester and a commercial microload test system (UNITECH). The electrical conductivity of stretchable conductors was measured by a four-point probe system. All results appearing in the text about mechanical properties and conductivity were based on average values of many $(>10)$ samples tested.

\section{References}

1. Ko, H. C. et al. A hemispherical electronic eye camera based on compressible silicon optoelectronics. Nature 454, 748-753 (2008).

2. Someya, T. et al. A large-area, flexible pressure sensor matrix with organic field effect transistors for artificial skin applications. Proc. Natl Acad. Sci. USA 101, 9966-9970 (2004).

3. Someya, T. et al. Conformable, flexible, large-area networks of pressure and thermal sensors with organic transistor active matrixes. Proc. Natl Acad. Sci. USA 102, 12321-12325 (2005).

4. Sekitani, T. et al. A large-area wireless power-transmission sheet using printed organic transistors and plastic MEMS switches. Nat. Mater. 6, 413-417 (2007).

5. Khang, D.- Y., Jiang, H., Huang, Y. \& Rogers, J. A. A stretchable form of singlecrystal silicon for high-performance electronics on rubber substrates. Science 311, 208-212 (2006).

6. Sekitani, T. et al. A rubberlike stretchable active matrix using elastic conductors. Science 321, 1468-1472 (2008).

7. Chun, K.- Y. et al. Highly conductive, printable and stretchable composite films of carbon nanotubes and silver. Nat. Nanotech. 5, 853-857 (2010).

8. Li, T., Huang, Z., Suo, Z., Lacour, S. P. \& Wagner, S. Stretchability of thin metal films on elastomer substrates. Appl. Phys. Lett. 85, 3435-3437 (2004).

9. Jeon, S. et al. Fabricating complex three-dimensional nanostructures with high-resolution conformable phase masks. Proc. Natl Acad. Sci. USA 101, 12428-12433 (2004).

10. Park, J. et al. Conformable solid-index phase masks composed of high-aspectRatio micropillar arrays and their application to 3D nanopatterning. Adv. Mater. 23, 860-864 (2011).

11. Park, J., Yoon, S., Kang, K. \& Jeon, S. Antireflection behavior of multidimensional nanostructures patterned using a conformable elastomeric phase mask in a single exposure step. Small 6, 1981-1985 (2010).

12. Jeon, S., Nam, Y.- S., Shir, D. J.- L \& Rogers, J. A. Three-dimensional nanoporous density graded materials formed by optical exposures through conformable phase masks. Appl. Phys. Lett. 89, 253101-253103 (2006).

13. Moon, J. H. \& Yang, S. Chemical aspects of three-dimensional photonic crystals. Chem. Rev. 110, 547-574 (2009).

14. Dentinger, P. M., Clift, W. M. \& Goods, S. H. Removal of SU-8 photoresist for thick film applications. Microelectron. Eng. 61-62, 993-1000 (2002). 
15. Jang, J. H., Ullal, C. K., Gorishnyy, T., Tsukruk, V. V. \& Thomas, E. L. Mechanically tunable three-dimensional elastomeric network/air structures via interference lithography. Nano Lett. 6, 740-743 (2006).

16. Moon, J. H., Ford, J. \& Yang, S. Fabricating three-dimensional polymeric photonic structures by multi-beam interference lithography. Polym. Adv. Technol. 17, 83-93 (2006).

17. Maldovan, M. \& Thomas, E. L. in Periodic Materials and Interference Lithography for Photonics Phononics and Mechanics 126-127 (Wiley-VCH, Weinheim, 2008).

18. Lee, J. N., Park, C. \& Whitesides, G. M. Solvent compatibility of Poly(dimethylsiloxane)-based microfluidic devices. Anal. Chem. 75, 6544-6554 (2003).

19. Jeon, S. et al. Molded transparent photopolymers and phase shift optics for fabricating three dimensional nanostructures. Opt. Express 15, 6358-6366 (2007).

20. Jeon, S., Malyarchuck, V., White, J. O. \& Rogers, J.A. Optically fabricated three-dimensional nanofluidic mixers for microfluidic devices. Nano Lett. 5 , 1351-1356 (2005).

21. Miao, L. et al. Thickness-dependent mechanical properties of polydimethyIsiloxane membranes. J. Micromech. Microeng. 19, 035028-035031 (2009).

22. Lötters, J. C. et al. The mechanical properties of the rubber elastic polymer polydimethylsiloxane for sensor applications. J. Micromech. Microeng. 7, 145-147 (1997).

23. Singamaneni, S. et al. Bifurcated mechanical behavior of deformed periodic porous solids. Adv. Funct. Mater. 19, 1426-1436 (2009).

24. Dickey, M. D. et al. Eutectic gallium-indium (EGaIn): A liquid metal alloy for the formation of stable structures in microchannels at room temperature. Adv. Funct. Mater. 18, 1097-1104 (2008).

25. Sekitani, T. et al. Stretchable active-matrix organic light-emitting diode display using printable elastic conductors. Nat. Mater. 8, 494-499 (2009).

26. Kramer, R. K., Majidi, C. \& Wood, R. J. Wearable Tactile Keypad with Stretchable Artificial Skin. IEEE Int. Conf. Robot. 1103-1107 (Shanghai, Chaina, 2011).

27. Cheng, S. \& Wu, Z. A microfluidic, reversibly stretchable, large-area wireless strain sensor. Adv. Funct. Mater. 21, 2282-2290 (2011).

28. Lipomi, D. J. et al. Skin-like pressure and strain sensors based on transparent elastic films of carbon nanotubes. Nat. Nanotech. 6, 788-792 (2011).

29. Kubo, M. et al. Stretchable microfluidic radiofrequency antennas. Adv. Mater 22, 2749-2752 (2010).

30. Kim, H.-J., Son, C. \& Ziaie, B. A multiaxiall stretchable interconnect using liquid-alloy-filled elastomeric microchannels. Appl. Phys. Lett. 92, 011904-011906 (2008)
31. So, J.-H. et al. Reversibly deformable and mechanically tunable fluidic antennas. Adv. Funct. Mater. 19, 3632-3637 (2009).

\section{Acknowledgements}

We thank the National Nanofab Center (NNFC) for fabricating silicon masters. This research was supported by WCU (World Class University) program through the National Research Foundation of Korea funded by the Ministry of Education, Science and Technology (R32-10051), the Pioneer Research Center Program through the National Research Foundation of Korea funded by the Ministry of Education, Science and Technology (20110001684), the Center for Advanced Soft Electronics under the Global Frontier Research Program through the National Research Foundation of Korea funded by the Ministry of Education, Science and Technology (20110031630). Y.H. and S.W. acknowledge support from the NSF grants ECCS0824129 and OISE-1043143. S.W. acknowledges support from the Ryan Fellowship and the Northwestern University International Institute for Nanotechnology. J.P. acknowledges support from the Global PhD. Fellowship through the National Research Foundation of Korea funded by the Ministry of Education, Science and Technology.

\section{Author contributions}

J.P. and S.J. conceived and designed the experiments. J.P. and C.A performed the experiments. J.P., D.S.K., and D.K.K. conducted mechanical characterization. S.W., M.L., and Y.H. conducted mechanics analysis. J.A.R. and S.J. initiated the idea for stretchable conductors. S.J. and J.A.R provided guidance. J.P., J.K.H. and S.J. wrote the manuscript with input from the other authors. All authors were involved in extensive discussions and data analysis.

\section{Additional information}

Supplementary Information accompanies this paper at http://www.nature.com/ naturecommunications

Competing financial interests: The authors declare no competing financial interests.

Reprints and permission information is available online at http://npg.nature.com/ reprintsandpermissions/

How to cite this article: Park, J. et al. Three-dimensional nanonetworks for giant stretchability in dielectrics and conductors. Nat. Commun. 3:916 doi: 10.1038/ncomms1929 (2012). 